\title{
The Electronic and Magnetic Properties of $\mathrm{UNiAs}_{2}$ Antiferromagnet
}

\author{
M. Werwiński*, A. Szajek, P. Leśniak And W.L. Malinowski \\ Institute of Molecular Physics, Polish Academy of Sciences, M. Smoluchowskiego 17, 60-179 Poznań, Poland \\ $\mathrm{UNiAs}_{2}$ band structure has been calculated based on two full-potential methods: full potential linearized \\ augmented plane wave implemented in WIEN2k code and full-potential local-orbital minimum-basis in FPLO \\ code. Starting from the local (spin) density approximation (L(S)DA) we verified either the orbital polarization \\ correction or the LSDA $+U$ approach with the Coulomb repulsion energies $U$ of $0-4 \mathrm{eV}$ for the uranium $5 f$ \\ electrons. Calculated magnetic moments confirm antiferromagnetic ground state and collinear magnetic sequence. \\ The best agreement with experimental results has been achieved by applying orbital polarization corrections, the \\ magnetic moment on uranium amounts to $1.76 \mu_{\mathrm{B}}$ per $\mathrm{U}$ atom.
}

PACS numbers: $71.20 .-\mathrm{j}$

\section{Introduction}

$\mathrm{UNiAs}_{2}[1]$ together with $\mathrm{UCuAs}_{2}[2], \mathrm{UPdAs}_{2}[3]$ and $\mathrm{UCoAs}_{2}[4]$ belong to a ternary arsenides group $\mathrm{UTAs}_{2}$, which is a part of a wider $\mathrm{UTX}_{2}$ family (where $\mathrm{X}=\mathrm{P}$, $\mathrm{As}, \mathrm{Sb}, \mathrm{Bi}$ ) [5, 6]. The wide range of properties in these ternary compounds is caused by the uranium $5 f$ electrons, which show an intermediate character between the localized $4 f$ and itinerant $3 d$ electrons system, $d$ electrons from transition metal $\mathrm{T}$ and $p$ electrons from a pnictide $\mathrm{X}$ are less significant. The crystallographic and magnetic data of $\mathrm{UNiAs}_{2}$ compound were reported by Fischer et al. [7]. Preliminary magnetic measurements have shown that compound orders antiferromagnetically at about $195 \mathrm{~K}$. The polycrystalline specimen has been the subject of neutron diffraction studies. It has been shown that magnetic moments are oriented along the $c$ direction and localized on uranium atoms. Measurements at $7.5 \mathrm{~K}$ yield $(1.85 \pm 0.06) \mu_{\mathrm{B}}$ with sequence +-+[5-7]. Previously, band structure for the isostructural $\mathrm{UCoAs}_{2}$ has been reported [8]. In this paper, we present the results of $a b$ initio electronic structure calculations designed to reproduce the magnetic properties of $\mathrm{UNiAs}_{2}$ compound.

\section{Details of calculations}

We used experimental lattice constants $(a=3.951 \AA$, $c=9.138 \AA$ ) and the Wyckoff positions [7]. The Wyckoff positions of uranium atoms were split into two nonequivalent sorts U1 and U2 (see Fig. 1 and Table I). This is to prepare unit cell for spin polarized calculations for

\footnotetext{
* corresponding author; e-mail: werwinski@ifmpan.poznan.pl
}

an antiferromagnet. This operation reduces symmetry from $P 4 / \mathrm{nmm}$ to $P 4 / \mathrm{mmm}$. The antiferromagnetic solution was not assumed in advance. In our calculations six inequivalent atoms forming unit cell were treated as different types. The starting magnetic moments on uranium atoms had opposite signs because of an initial spin splitting. It allowed the system to reach antiferromagnetically ordered ground state. Calculations were performed by using the WIEN2k [9] and the FPLO [10] codes, which are based on the density functional theory (DFT).

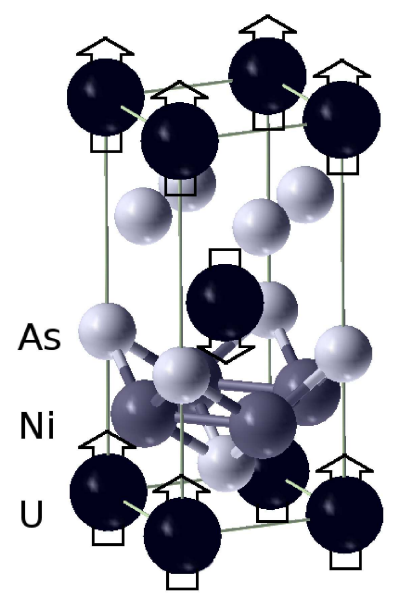

Fig. 1. The crystallographic and magnetic structure of $\mathrm{UNiAs}_{2}$.

\section{WIEN2k}

We used the full-potential linearized augmented plane-wave method (FP-LAPW) as implemented in the 
TABLE I

The atomic positions of $\mathrm{UNiAs}_{2}$. The antiferromagnetic unit cell data are based on the experimental crystallographic data [7].

\begin{tabular}{c|c|c|c}
\hline \hline Atom & $x$ & $y$ & $z$ \\
\hline $\mathrm{U} 1$ & 0.0 & 0.0 & 0.0 \\
$\mathrm{U} 2$ & $1 / 2$ & $1 / 2$ & 0.5088 \\
$\mathrm{Ni}$ & 0.0 & $1 / 2$ & 0.2544 \\
$\mathrm{As} 1$ & 0.0 & 0.0 & 0.4075 \\
$\mathrm{As} 2$ & $1 / 2$ & $1 / 2$ & 0.1013 \\
As3 & $1 / 2$ & 0.0 & 0.7544
\end{tabular}

WIEN2k code within the generalized gradient approximation with the spin-orbit interaction $(\mathrm{GGA}+\mathrm{SO})$ for the exchange-correlation potential. The exchange-correlation potential was used in the Perdew, Burke, Ernzerhof form PBE96 [11]. Calculations were performed for $1001 k$-points in the irreducible wedge of the Brillouin zone $(26 \times 26 \times 11 \mathrm{mesh})$ and a plane wave cut-off parameter of $R K_{\mathrm{MAX}}=8$. The total energy convergence criterion was $10^{-6} \mathrm{Ry}$.

The LSDA $+U$ method introduced by Anisimov et al. [12] was used to better describe strong correlations.

Taking into account the so-called orbital polarization (OP) term as proposed by Brooks [13] and Eriksson et al. [14] is another approach to improve discrepancy between calculated and experimental magnetic moment.

\section{FPLO}

The spin polarized fully relativistic calculations were carried out based on the full-potential local-orbital minimum-basis scheme FPLO (FPLO-5.10-20 and FPLO-7.00) $[10,15]$. The calculations were performed for the reciprocal space mesh containing 1155 irreducible $k$-points from $7436(26 \times 26 \times 11)$ within the irreducible wedge of the Brillouin zone using the tetrahedron method for integrations [16]. The LSDA exchange-correlation potential was used in the Perdew and Wang form PW92 [17]. Computations were done with and without orbital polarization corrections $[18,19]$. The self-consistent criterion was equal to $10^{-6} \mathrm{Ry}$ for the total energy.

\section{Results and discussion}

The spin polarized calculations were performed for a different initial spin splitting. Both ferro- and antiferromagnetic solutions were obtained, but the latter was more stable by about $0.05 \mathrm{eV}$ per unit cell (for the WIEN2k calculations).

Figures 2 and 3 present calculated densities of electronic states (DOS) obtained based on the WIEN2k and

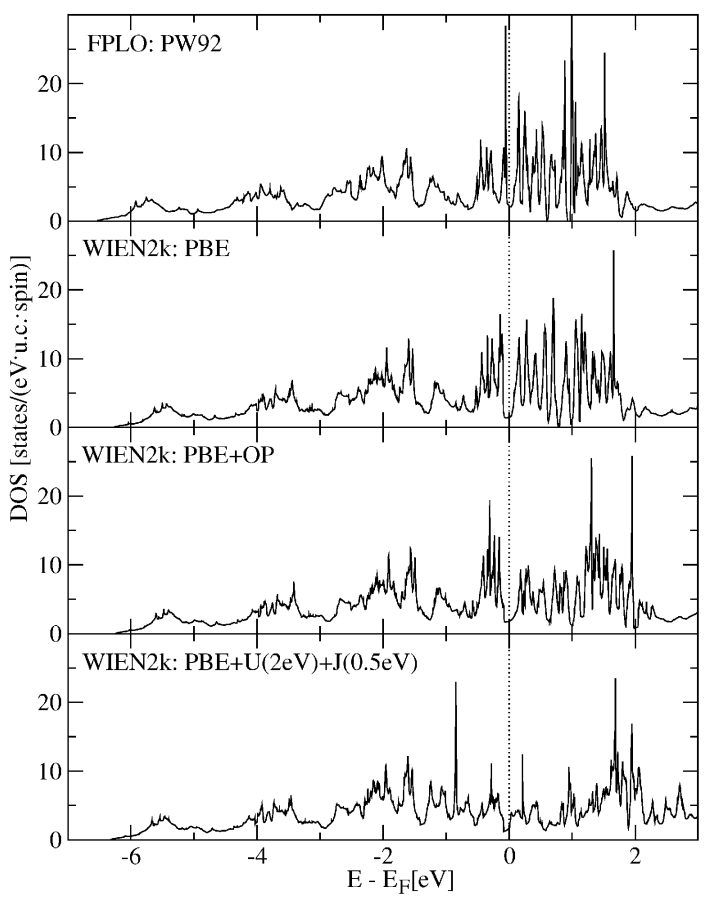

Fig. 2. Total densities of states calculated without and with orbital polarization corrections and using the $\mathrm{LDA}+U(\mathrm{GGA}+U)$ formalism.

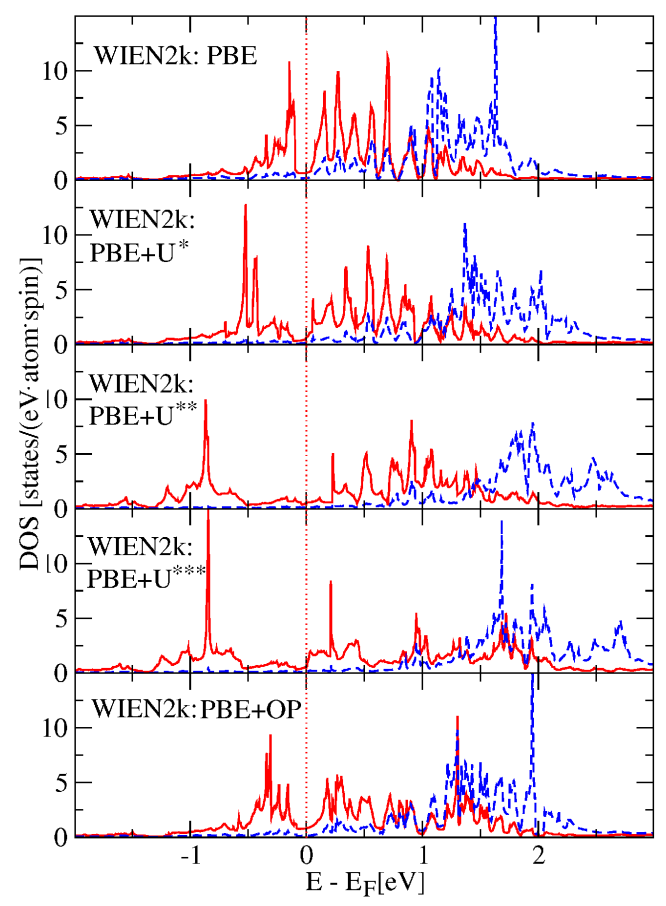

Fig. 3. Spin projected partial densities of states for uranium (U1) $5 f$-electrons calculated without and with orbital polarization corrections and using the $\mathrm{LDA}+U$ $(\mathrm{GGA}+U)$ formalism. Solid blue curves for the majority spin and dash red curves for minority spin. $\mathrm{PBE}+U^{*}$ for $U=1 \mathrm{eV} ; \mathrm{PBE}+U^{* *}$ for $U=2 \mathrm{eV} ; \mathrm{PBE}+U^{* * *}$ for $U=2 \mathrm{eV}$ and $J=0.5 \mathrm{eV}$ 
TABLE II

Spin projected total and partial densities of states on the Fermi level [states/(eV (unit cell or atom) spin)] and the Sommerfeld specific heat coefficient $\gamma\left[\mathrm{mJ} /\left(\mathrm{mol} \mathrm{K}^{2}\right)\right]$ obtained from the calculations based on the WIEN2k code.

\begin{tabular}{l|c|c|c|c}
\hline \hline & Total & $\mathrm{U} 1(5 f)$ & $\mathrm{U} 2(5 f)$ & $\gamma$ \\
\hline $\mathrm{PBE}$ & 1.45 & 0.67 & 0.20 & 3.42 \\
$\mathrm{PBE}+U(1 \mathrm{eV})$ & 1.53 & 0.61 & 0.16 & 3.61 \\
$\mathrm{PBE}+U(2 \mathrm{eV})$ & 1.84 & 0.49 & 0.13 & 4.34 \\
$\mathrm{PBE}+U(2 \mathrm{eV})+J(0.5 \mathrm{eV})$ & 1.76 & 0.56 & 0.12 & 4.15 \\
$\mathrm{PBE}+\mathrm{OP}$ & 1.78 & 0.82 & 0.15 & 4.19
\end{tabular}

the FPLO methods. The valence bands plotted in Fig. 2 have similar width. The Fermi level $\left(E_{\mathrm{F}}\right)$ is located on a pseudogap. Values of DOS at the Fermi level are collected in Table II. The main contribution to DOS in the vicinity of $E_{\mathrm{F}}$ is due to the $\mathrm{U} 5 f$ electrons which provide about $60 \%$ of the total value. The spin projected DOS plots of U $5 f$ electrons are collected in Fig. 3. Unfortunately, to our best knowledge, the experimental value of the Sommerfeld coefficient is not available so we are not able to make a comparison.

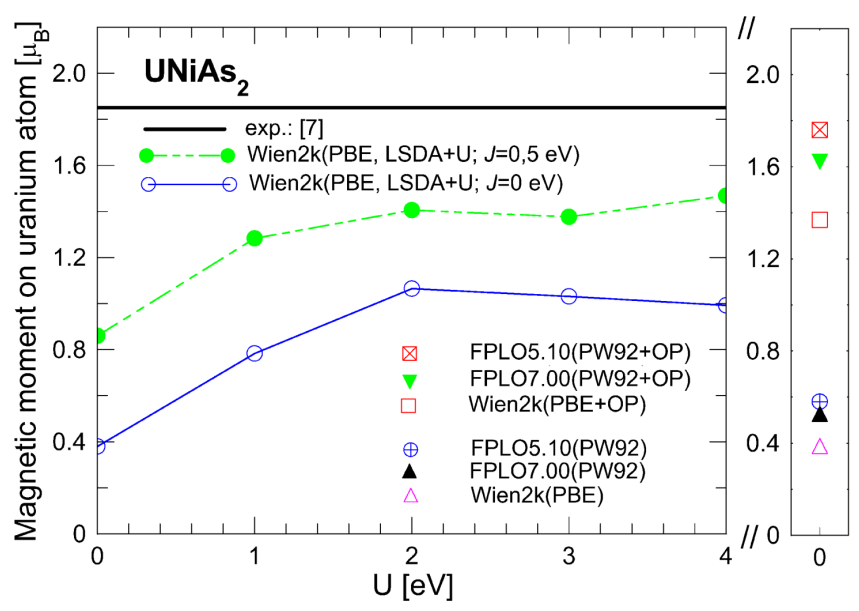

Fig. 4. The magnetic moment on uranium atom.

TABLE III

The spin, orbital and total local magnetic moments $\mu\left[\mu_{\mathrm{B}}\right]$ on U1 atom obtained from calculations based on the codes: WIEN2k (PBE) and FPLO7 (PW92). The magnetic moments on U2 atom are exactly opposite.

\begin{tabular}{l|c|c|c}
\hline \hline Method & $\mu_{s}$ & $\mu_{l}$ & $\mu_{\text {tot }}$ \\
\hline WIEN2k PBE & 1.48 & -1.86 & -0.38 \\
FPLO-7 PW92 & 1.38 & -1.96 & -0.58 \\
WIEN2k PBE $+U=2 \mathrm{eV}, J=0.5 \mathrm{eV}$ & 1.94 & -3.35 & -1.41 \\
FPLO-5.10 PW92+OP & 1.64 & -3.40 & -1.76
\end{tabular}

The LSDA $+U$ procedure causes shift of occupied $U 5 f$ states to higher binding energies (to about $2 \mathrm{eV}$ below $E_{\mathrm{F}}$ for $\left.U=2 \mathrm{eV}\right)$. The $\mathrm{U} 5 f$ states occupied by electrons with opposite spin are shifted towards higher energies above $E_{\mathrm{F}}$. The experimental value of magnetic moments $1.85 \mu_{\mathrm{B}}$ on uranium atoms is known from neutron diffraction experiment [7]. Both methods, WIEN2k and FPLO, within the LSDA approach gave underestimated values between 0.38 and $0.58 \mu_{\mathrm{B}} / \mathrm{U}$ atom. Such discrepancy is usually caused by a wrong treatment of correlation effects within the LSDA. Two series of calculations within the $\mathrm{LSDA}+U$ approach were performed to improve the description of $5 \mathrm{f}$ electrons. On site Coulomb energy $U$ was varied from 0 to $4 \mathrm{eV}$ for the exchange interaction term $J$ equal to 0 and $0.5 \mathrm{eV}$ (see Fig. 4). The magnetic moment saturates above $U=2 \mathrm{eV}$ for both series, but values are far from the experimental one. It is well-known fact that orbital moments calculated within the LSDA are too small. Using the so-called orbital polarization formalism one can overcome this drawback. Calculated magnetic moments are collected in Fig. 4 and Table III. The OP formalism improve results. Especially values obtained by two versions of the FPLO code are close to the experimental one.

\section{Summary and conclusions}

In this paper we presented results of the $a b$ initio band structure calculations for the $\mathrm{UNiAs}_{2}$ compound. Two methods, the FP-LAPW and the FPLO, were used within the LSDA, LSDA $+U$, and LSDA + OP formalism. The spin polarized calculations reproduced an antiferromagnetic solution with local magnetic moments on uranium atoms. A good agreement with an experimental result was obtained applying the $\mathrm{LSDA}+\mathrm{OP}$ formalism.

\section{Acknowledgments}

This work was supported by funds for science in years 2007-2010 as a research projects no. N N202 134933 and N N202 288338.

\section{References}

[1] Z. Żołnierek, D. Kaczorowski, R. Troć, J. Less Common Met. 121, 193 (1986).

[2] J. Stępień-Damm, D. Kaczorowski, R. Troć, J. Less Common Met. 132, 15 (1987).

[3] A. Murasik, P. Fischer, D. Kaczorowski, J. Phys., Condens. Matter 2, 3967 (1990).

[4] D. Kaczorowski, H. Noël, M. Potel, J. Alloys Comp. 302, 1 (2000).

[5] D. Kaczorowski, J. Alloys Comp. 186, 333 (1992).

[6] D. Kaczorowski, R. Kruk, J.P. Sanchez, B. Malaman, F. Wastin, Phys. Rev. B 58, 9227 (1998).

[7] P. Fischer, A. Murasik, D. Kaczorowski, R. Troć, Physica B 156, 829 (1989).

[8] M. Werwiński, A. Szajek, Acta Phys. Pol. A 115, 244 (2009). 
[9] P. Blaha, et al., WIEN2k 7.3, An Augmented Plane Wave + Local Orbitals Program for Calculating Crystal Properties, Karlheinz Schwarz, Techn. Universität Wien, Austria, 2007.

[10] FPLO code by K. Koepernik, H. Eschrig, Phys. Rev. B 59, 1743 (1999); http://www.fplo.de .

[11] J.P. Perdew, K. Burke, M. Ernzerhof, Phys. Rev. Lett. 77, 3865 (1996).

[12] V.I. Anisimov, I.V. Solovyev, M.A. Korotin, M.T. Czyzyk, G.A. Sawatzky, Phys. Rev. B 48, 16929 (1993).

[13] M.S.S. Brooks, Physica B 130, 6 (1985).

[14] O. Eriksson, B. Johansson, M.S.S. Brooks, J. Phys. C 1, 4005 (1989).
[15] M. Richter, K. Koepernik, H. Eschrig, in: 43rd Karpacz Winter School of Theoretical Physics, Ladek Zdrój (Poland) 2007, Ed. J. Jędrzejewski, World Sci., Singapore 2008, p. 271.

[16] P. Blöchl, O. Jepsen, O.K. Andersen, Phys. Rev. B 49, 16223 (1994)

[17] J.P. Perdew, Y. Wang, Phys. Rev. B 45, 13244 (1992).

[18] O. Eriksson, M.S.S. Brooks, B. Johansson, Phys. Rev. B 41, 7311 (1990).

[19] I. Opahle, K. Koepernik, H. Eschrig, Phys. Rev. B 60, 14035 (1999). 